BMJ Open

Sport \&

Exercise

Medicine

\title{
Movement pattern training compared with standard strengthening and flexibility among patients with hip- related groin pain: results of a pilot multicentre randomised clinical trial
}

\author{
Marcie Harris-Hayes (D) , ${ }^{1,2}$ Karen Steger-May, ${ }^{3}$ Allyn M Bove, ${ }^{4}$ Stefanie N Foster, ${ }^{5}$ \\ Michael J Mueller, ${ }^{5}$ John C Clohisy, ${ }^{6}$ G Kelley Fitzgerald ${ }^{4}$
}

To cite: Harris-Hayes M, Steger-May K, Bove AM, et al. Movement pattern training compared with standard strengthening and flexibility among patients with hiprelated groin pain: results of a pilot multicentre randomised clinical trial. BMJ Open Sport \& Exercise Medicine 2020;6:e000707. doi:10.1136/ bmjsem-2019-000707

- Additional material is published online only. To view please visit the journal online (http://dx.doi.org/10.1136/ bmjsem-2019-000707).

Accepted 8 March 2020
Check for updates

C Author(s) (or their employer(s)) 2020. Re-use permitted under CC BY-NC. No commercial re-use. See rights and permissions. Published by BMJ.

For numbered affiliations see end of article.

Correspondence to Dr Marcie Harris-Hayes; harrisma@wustl.edu

\section{ABSTRACT \\ Study design Pilot, multicentre randomised clinical trial (RCT). \\ Objectives Assess viability of performing a definitive RCT and compare preliminary effects of movement pattern training (MoveTrain) and strengthening/flexibility (Standard) to improve function in people with chronic hip-related groin pain (HRGP).}

Background To determine the best physical therapistled intervention for patients with HRGP, we must understand treatment effects of different treatment modes.

Methods Forty-six patients (17M:29F; $29 \pm 5.3$ years; body mass index $25.6 \pm 6.3 \mathrm{~kg} / \mathrm{m}^{2}$ ) with HRGP were randomised. MoveTrain included task-specific training to optimise biomechanics during daily tasks. Standard included strengthening/flexibility. Treatment included 10 visits/12 weeks and home exercise programme (HEP). Primary outcomes for feasibility were recruitment, retention, treatment adherence and treatment fidelity. Secondary outcomes were patient-reported function (Hip disability and Osteoarthritis Outcome Score (H0OS)), lower extremity kinematics and hip muscle strength.

Results We achieved target recruitment, and retention was excellent (91\%). Patient session attendance was high (93\%); however, reported HEP adherence (62\%) was lower than expected. Physical therapists' adherence to treatment protocols was high (90\%). Patients demonstrated high treatment receipt; $91 \%$ of exercises performed were rated independent. Both groups demonstrated clinically important improvements in function (HOOS) and muscle strength; however, there were no between-group differences (HOOS subscales, $p \geq 0.13$, strength, $p \geq 0.34$ ). Compared with Standard, MoveTrain demonstrated greater reductions in hip adduction $(\mathrm{p}=0.016)$ and pelvic drop $(p=0.026)$ during a single leg squat. No adverse events were noted.

Conclusion Our experience in completing this RCT confirmed that a larger, multicentre RCT is feasible and highlighted modifications we will implement to optimise the future RCT.

Trial registration number NCT02913222.

\section{What are the new findings}

Using our methods, a large, multicentre randomised clinical trial (RCT) to compare MoveTrain and Standard treatment for patients with hip-related groin pain (HRGP) is feasible as demonstrated by high retention, patient adherence and treatment fidelity.

- Preliminary findings related to patient-reported outcomes, kinematics and strength suggest that rehabilitation may be appropriate for patients with HRGP however, further study is needed

- A better understanding of treatment effects of rehabilitation will allow us to better compare surgical and non-surgical treatments, thus ultimately leading to the ability to better match patients to treatment.

\section{INTRODUCTION}

Hip-related groin pain (HRGP) results in significant pain and activity limitations among young to middle aged adults. Hip disorders proposed to contribute to HRGP include femoroacetabular impingement syndrome (FAIS), acetabular dysplasia, labral tears and injury to other intra-articular structures. Additionally, acetabular dysplasia and FAIS are associated with development of hip osteoarthritis. ${ }^{12}$ It is important to intervene early and provide effective treatment to improve pain and function among those with HRGP and potentially prevent or delay hip osteoarthritis (OA).

While significant literature exists related to surgical interventions, evidence related to physical therapist (PT)-led intervention remains sparse. ${ }^{3-5}$ Three randomised clinical trials (RCTs) were recently published comparing arthroscopic surgery to PT-led intervention for patients with FAIS. Two studies ${ }^{6}$ reported greater improvement among those who received surgery compared 
with PT-led intervention, but one study reported no differences between the two interventions. ${ }^{8}$ These mixed findings may be partially due to limited evidence available to inform PT-led intervention. PT-led interventions described in previous RCTs were based primarily on expert opinion, resulting in intervention that is multimodal and non-standard, thus limiting our ability to make conclusions about the effect of any one treatment. To ultimately determine comparative effectiveness of PT-led intervention and surgical intervention of HRGP, we must first determine the best PT-led intervention by understanding the effects of different treatments. We compared two PT-led interventions, movement pattern training (MoveTrain) and traditional strengthening and flexibility (Standard).

The goal of MoveTrain is to reduce hip joint stress by optimising biomechanics during functional tasks. The key element of MoveTrain is task-specific instruction to correct abnormal movement patterns during daily activities. In our previous proof-of-concept study, we noted that patients with HRGP who participated in MoveTrain were able to reduce hip adduction motion during a single leg squat, ${ }^{9}$ and this reduction was associated with improved patient-reported function. ${ }^{9}{ }^{10}$ Given resources required to conduct a larger RCT to definitively determine MoveTrain effects, we designed a pilot, multicentre RCT to assess the viability of performing a definitive RCT.

The primary purpose of this study was to determine patient retention and treatment adherence and to demonstrate treatment fidelity among treatment providers and patients at both clinical sites. Our secondary purpose was to compare the preliminary effects of MoveTrain to Standard on patient-reported function, lower extremity kinematics (targeted by MoveTrain) and hip muscle strength (targeted by Standard).

\section{METHODS}

\section{Study design, setting and patient involvement}

This study was a pilot, multicentre RCT (figure 1). Data collection and treatment occurred at The Movement Science Research Center at Washington University's Program in Physical Therapy and The Physical Therapy - Clinical and Translational Research Center at the University of Pittsburgh. Washington University served as the coordinating centre and single site institutional review board (IRB). This study was approved by Human Research Protection Offices of Washington University and University of Pittsburgh. All patients signed an informed consent statement prior to participating. Patients were not involved in the design, recruitment or conduct of the study.

\section{Manual of operations (MOP) development and training}

An MOP was developed to document standard methods for all study components. To optimise treatment fidelity, we used the treatment fidelity framework developed by National Institutes of Health's Behavioral Change Consortium. ${ }^{11}{ }^{12}$ Active ingredients believed to affect patient outcomes for each treatment arm were identified and operationally defined. Each treatment protocol was reviewed by experts at both sites to ensure it reflected the underlying theory of its respective approach. The first author led onsite training for examiners $(n=2)$ and treating PTs $(n=5)$; all were PTs with 6-27 years of experience. Training included review and discussion of the MOP, patient scenario role plays to demonstrate assessment and treatment concepts and discussion to identify barriers to study protocol performance and patient treatment adherence. Training time totalled 16 hours per site.

\section{Participants}

Patients were recruited between January 2017 and February 2018, from healthcare clinics; research volunteer databases; social media; and other written communications. To be eligible, patients had to be 15-40 years old; report deep hip joint or anterior groin pain that was reproduced with flexion, adduction, internal rotation impingement test ${ }^{13}$; report pain $\geq 3 / 10$ and present $\geq 3$ months; and demonstrate functional limitation with modified Harris Hip Score ${ }^{14}<90$. Exclusion criteria included previous hip surgery, fracture, infection or pain due to high impact trauma; diagnosed with Legg-Calve-Perthes disease or slipped capital femoral epiphysis; inflammatory disease; neurological involvement affecting balance; pain, numbness or tingling that radiates into thigh; pregnancy; and screening tests indicating hip pain was referred from the spine.

\section{Assessment (baseline and post-treatment)}

Patients who passed initial screening were scheduled for clinical examination, which included assessment of final screening criteria and, if the patient was determined to be eligible, baseline assessment. Patients completed selfreport questionnaires and participated in assessment of movement patterns and hip strength. Patients were then randomised into MoveTrain or Standard. Thirteen weeks after enrolment, patients returned for post-treatment testing.

\section{Treatments}

Treatments were delivered according to the Template for intervention Description and Replication Guidelines. ${ }^{15}$ Treatment for both groups included 10 supervised sessions over 12 weeks and daily home exercise programme (HEP). Both treatment arms included assessment of patient goals, patient education and HEP instruction. Patient education focused on patient-specific tasks, which were identified using the Patient Specific Functional Scale (PSFS). ${ }^{16}$ A brief description of each treatment arm is provided below.

Movement pattern training (MoveTrain) (online supplementary appendix A): treatment focus was on taskspecific training to improve lower extremity kinematics during functional and patient-specific tasks. Patient education included instruction in abnormal movement patterns and methods to optimise movement patterns 


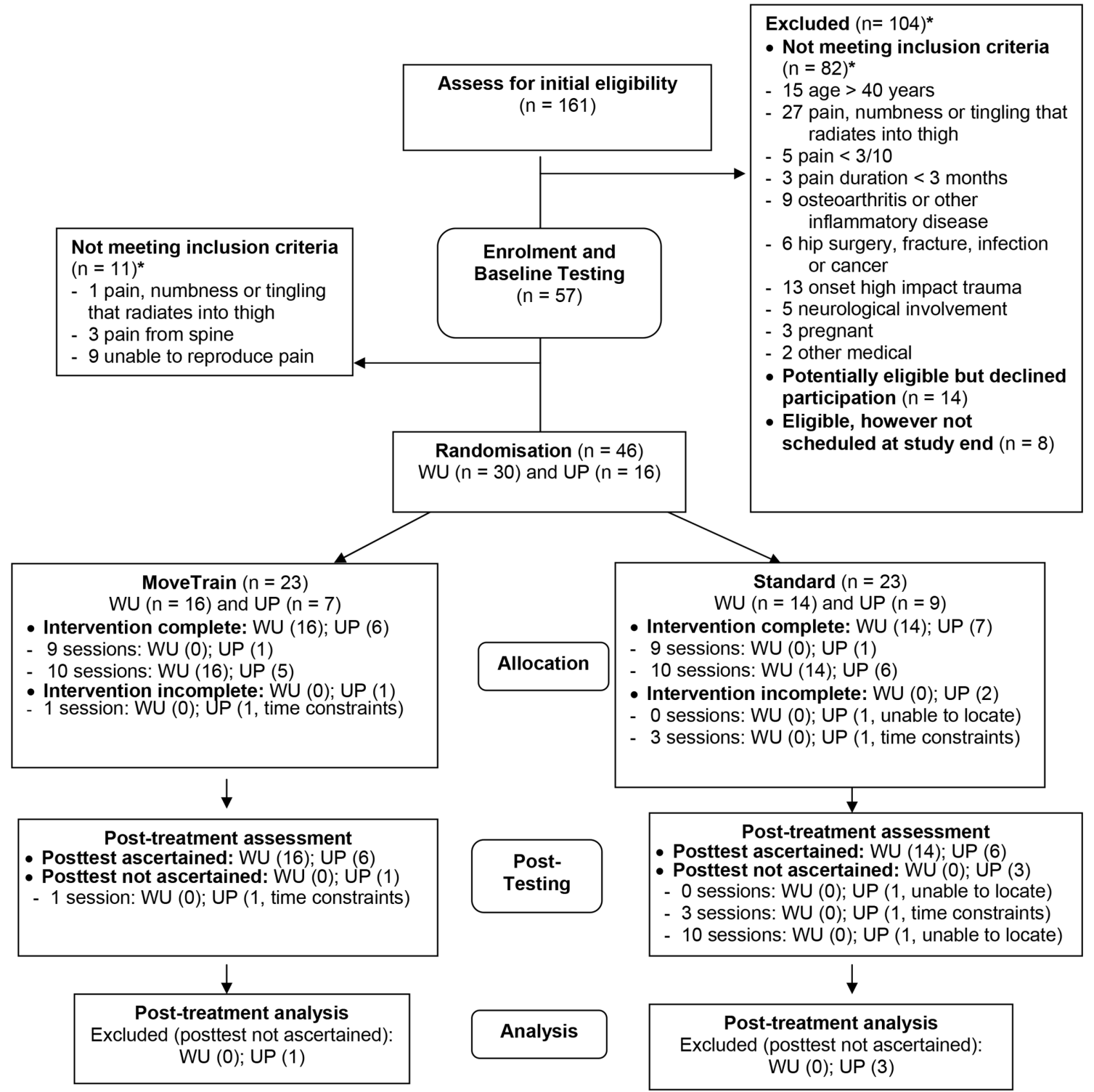

Figure 1 Study flow diagram. CONSORT flow diagram adapted. CONSORT, Consolidated Standards of Reporting Trials; WU, Washington University; UP, University of Pittsburgh. *inclusion criteria categories are not mutually exclusive.

during each task. Exercises included repeated practice of tasks using optimised movement patterns. Verbal cues and visual aids were used to assist the patient. Based on patient performance, task difficulty was progressed by varying repetitions performed, increasing load or speed or changing support surface.

Standard rehabilitation (Standard) (online supplementary appendix B): treatment focus was on progressive lower extremity and trunk strengthening and lower extremity flexibility. Patient education included instruction to modify intensity, frequency or duration of patient-specific tasks. Strengthening and flexibility exercises prescribed were selected using current clinical practice guidelines ${ }^{17}$ and previous reports. ${ }^{518}$ Each patient was progressed by increasing repetitions performed or increasing load.

\section{Primary outcomes: feasibility}

Primary outcomes were related to feasibility of the larger trial. Recruitment rate was defined as the number of patients deemed eligible who agreed to participation. Retention rate was defined as the percentage of those who completed post-treatment testing. Patient treatment session adherence was defined as the percentage of supervised visits attended. Patient HEP adherence was defined as the percentage of days the patient reported completing their HEP.

To assess treatment fidelity, ${ }^{11} 12$ we assessed PT treatment delivery and patient treatment receipt. Treatment delivery was assessed using prestudy assessment and poststudy chart reviews to assess protocol adherence. Prestudy assessment included a written exam and roleplayed scenarios. ${ }^{19}$ For role-played scenarios, a checklist was used to document standard performance of active ingredients for each treatment. To assess protocol adherence, a PT who was not involved in providing treatment completed chart reviews. A standard form was used to identify if performance of active ingredients for each 
treatment arm was documented. Protocol adherence was scored as percentage of active ingredients completed.

In addition to our a prior feasibility outcomes, we assessed patient treatment receipt, which relates to ability to understand key treatment concepts, and ability to independently perform tasks. ${ }^{11} 12$ Using our previously published methods, ${ }^{19}$ treatment receipt was scored by the treating PT as the proportion of exercises the patient performed independently.

\section{Secondary outcomes: preliminary effectiveness}

Preliminary effectiveness outcomes determined a priori were the Hip dysfunction and Osteoarthritis Outcome Score (HOOS) subscales of activities of daily living (ADL) and symptoms, ${ }^{20}$ hip adduction angle during a single leg squat and hip muscle strength.

\section{Patient-reported outcome measures}

The HOOS is a reliable ${ }^{21-23}$ and valid ${ }^{2123}$ hip-specific patient-reported outcome used to quantify activity limitations among those with hip disorders. The values for the HOOS subscales, including pain, symptoms, ADLs, sport and recreation (Sport) and quality of life range from 0 to 100 with higher scores indicating higher levels physical function. The established values for minimum important change (MIC) for people with HRGP who have undergone arthroscopic surgery are 8, 9, 9, 6, 10 and 11 , respectively. ${ }^{23}$ Values for MIC for non-surgical management are not available. In addition, we collected PSFS $^{16}$; Patient-Reported Outcomes Measurement Information System (PROMIS) Scores ${ }^{24}$; and Pain Numeric Rating Scale. ${ }^{26}$ Information for the additional measures are provided in table 4.

\section{Kinematics}

A digital camera was used to capture two-dimensional motion while the patient completed three single leg squats. $^{27}$ figure 2 demonstrates the variables of interest including hip adduction, pelvic tilt (obliquity/drop) and trunk lean (ipsilateral lean). Values of three trials were averaged for each variable. Inter-rater reliability of all measures among our group was excellent (intraclass correlation $\left(\mathrm{ICC}_{3,3:}\right)>0.98$, SE of measurements $<1^{\circ}$ ).

\section{Muscle strength}

A microFET3 hand-held dynamometer (Hogan Health Industries, Salt Lake City, Utah, USA) was used to assess hip muscle strength of hip external rotators, internal rotators, abductors, adductors, flexors and extensors. Make tests ${ }^{28}$ were used, incorporating a strap for resistance. After a practice trial, three maximal trials were performed. Force values of three trials were averaged and used to calculate torque, then normalised by weight and height. ${ }^{29}$ Strength was not a primary outcome, and this study was funded by an R21 mechanism, which provides limited resources. Performing an inter-rater reliability

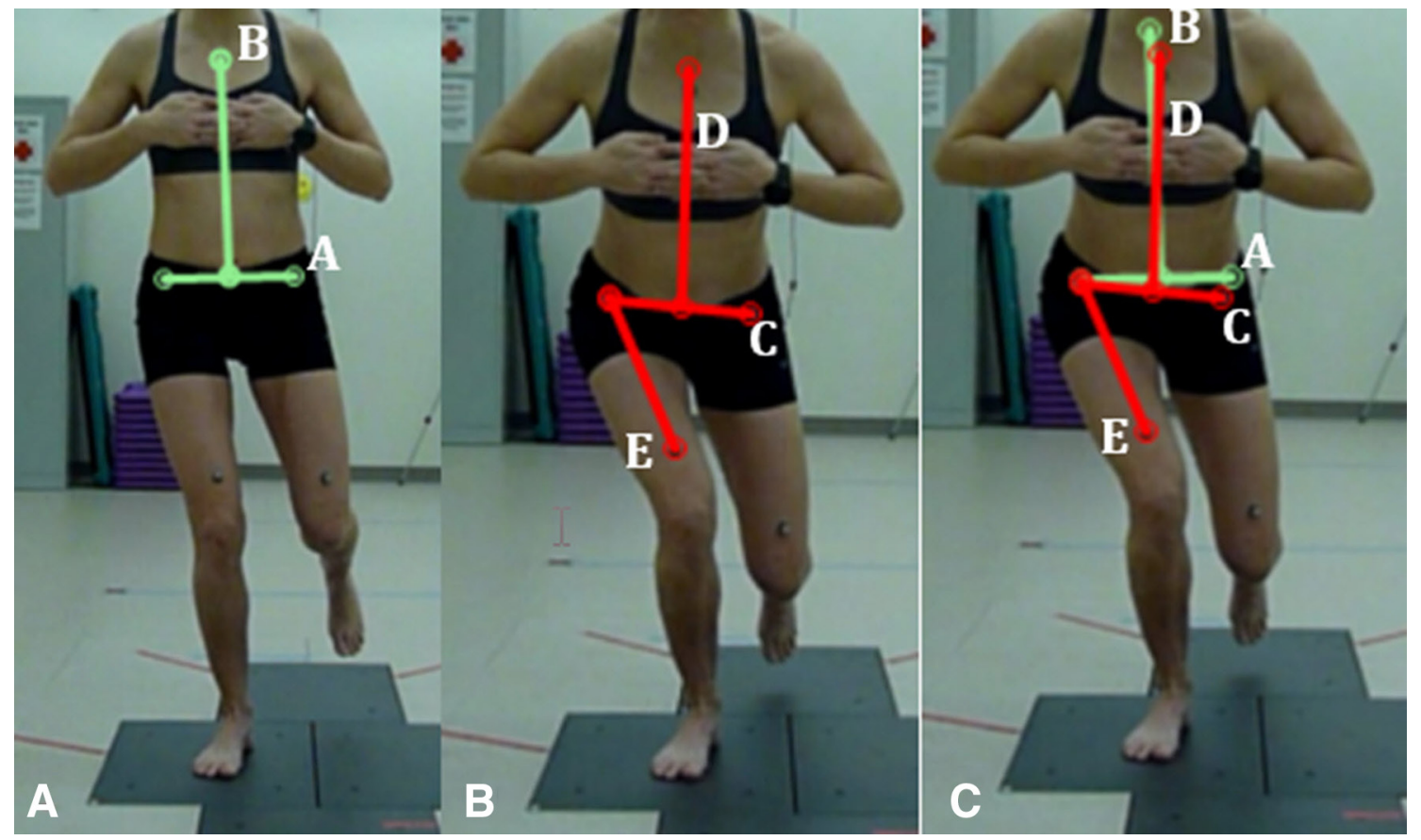

Figure 2 (A) Initial position. Line A is drawn between the anterior superior iliac spine (ASIS) markers and is the initial position of the pelvis. Line $B$ is drawn from the sternal marker to the midpoint of line $A$ and is the initial position of the trunk. (B) Final depth of squat. Line C is drawn between the ASIS markers and is the final position of the pelvis. Line D is drawn from the sternal marker to the midpoint of line $\mathrm{C}$ and is the final position of the trunk. Line $\mathrm{E}$ is drawn from the ASIS marker to the femur marker on the weight-bearing leg and is the final position of the femur. (C) Angle measurement. Hip adduction angle is defined by lines $\mathrm{C}$ and $\mathrm{E}$. Positive values indicate hip adduction; pelvic excursion is defined by lines $\mathrm{A}$ and $\mathrm{C}$. Negative values indicate a pelvic tilt in which the non-weight-bearing side is lowering; trunk excursion is defined by lines $B$ and $D$. Positive values indicate the trunk is leaning towards the weightbearing limb. 
study of each secondary outcome was simply not feasible within this mechanism. A previous study using similar methods reported high test-retest reliability, ICCs $>0.82$.

\section{A priori criteria for success}

A priori criteria for success included: (1) 46 participants recruited; (2) 90\% retention ${ }^{9}$; (3) $90 \%$ adherence to attending treatment sessions; (4) $80 \%$ adherence to HEP; and (5) treatment providers scoring at least $95 \%$ on prestudy examination and $80 \%$ protocol adherence.

We hypothesised MoveTrain would demonstrate greater improvement in HOOS and greater reduction in peak hip adduction during functional tasks compared with Standard, despite possibly demonstrating less hip strength.

\section{Pilot trial sample size}

Sample size for primary outcomes was based on precision and assumed that observed rates of adherence and retention would be at least $90 \% .{ }^{9}$ With a sample size of 46 (23/group), 95\% CI around observed rates of adherence and retention were expected to be $78 \%-96 \%$ (ie, within $9 \%$ of the true rate). The study was designed to estimate effect sizes for treatment outcomes; therefore, the study was $80 \%$ powered to detect large effect sizes of 0.9 or above.

\section{Randomisation}

Patients were randomised to treatment in 1:1 ratio stratified by site, sex and HOOSSymptoms subgroup (determined from median split of preliminary data). Within each stratum, patients were allocated using a variable block size. Randomisation sequences were generated a priori using a formal probability model and were elicited from the data capture system. Given the nature of treatment, it was not possible to blind treating PTs or patients to treatment assignment. Study personnel performing assessments and kinematic measurements were blinded to group.

\section{Statistical analysis}

Fisher's exact test compared retention and patient adherence to treatment session rates by site and by group. Patient adherence to HEP, therapists' treatment delivery and patient treatment receipt were compared by site and group using Wilcoxon's test.

Data collected at pretest and post-test were analysed with analysis of covariance where post-test was the dependent variable, pretest was the covariate and treatment group was the independent variable that tests the null hypothesis that after adjusting for pretest, post-test is not significantly different across treatment groups. A general linear model was used when residuals were normally distributed, and homogeneity of regression lines assumption was satisfied, otherwise non-parametric covariance analysis $^{30}$ was used. Multinomial generalised estimating equations was used to analyse ordinal pain numeric rating scales.

\section{RESULTS}

\section{Primary outcomes: feasibility}

Table 1 provides baseline characteristics of study groups. Overall results related to study feasibility along with sitespecific and treatment group-specific comparisons are provided in table 2.

\section{Recruitment and Retention Rate}

A flow diagram of patient recruitment and retention are provided in figure 1. Overall, 42/46 (91\%; 95\% CI 83\% to $99 \%$ ) of patients completed post-treatment testing (table 2).

\section{Treatment adherence}

Overall, $43 / 46$ (93\%; $95 \%$ CI $86 \%$ to $100 \%)$ of patients attended at least $90 \%$ of their sessions. The 43 patients who completed at least $90 \%$ of supervised sessions reported HEP completion on 62\% (range 9\%-97\%) of days.

\section{PTs' treatment delivery}

All providers scored $>95 \%$ on written exam and roleplayed scenarios. Chart reviews resulted in data for 132 treatment visits for the 45 patients who completed at least one treatment visit. Overall, the proportion of active ingredients completed by PTs was 90\% (range $60 \%-100 \%$ ).

\section{Patients' treatment receipt}

Overall, the proportion of exercises rated independent was $91 \%$ (range $18 \%-100 \%$ ).

\section{Protocol deviations}

We had nine protocol deviations. Two patients had their baseline HOOSSymptom score entered into the data system incorrectly, resulting in each patient being randomised to treatment within an incorrect stratum. Due to personal scheduling issues, four patients completed post-treatment examination before 13 weeks and three completed post-treatment examination after 16 weeks.

\section{Safety}

No unexpected adverse events were noted. Twenty-seven patients reported discomfort in hip, knee or spine with performance of at least one exercise (table 3). More patients in Standard reported pain with at least one exercise compared with MoveTrain ( $85 \%$ vs $45 \%$, respectively $\mathrm{p}=0.008$ ). Each reported exercise was modified by the treating PT.

\section{Secondary outcomes: preliminary effectiveness}

Both groups demonstrated improvements in all HOOS subscales; however, there were no between-group differences (table 4). Both groups also demonstrated similar improvements in PSFS, PROMIS scores and Pain Numeric Rating scales. MoveTrain demonstrated a greater reduction in hip adduction and pelvic tilt (drop) during the single leg squat compared with Standard (table 5). Both groups demonstrated similar 
Table 1 Demographics for all enrolled patients and patients who provided post-test data by treatment group $(n=46)$

\begin{tabular}{|c|c|c|c|c|}
\hline \multirow[b]{2}{*}{ Variable } & \multirow{2}{*}{$\begin{array}{l}\text { All enrolled } \\
\text { Patients } \\
\mathrm{n}=46\end{array}$} & \multicolumn{3}{|c|}{ Patients providing post-test data, by treatment group } \\
\hline & & $\begin{array}{l}\text { All } \\
\mathrm{n}=42\end{array}$ & $\begin{array}{l}\text { MoveTrain } \\
\mathrm{n}=22\end{array}$ & $\begin{array}{l}\text { Standard } \\
\mathrm{n}=20\end{array}$ \\
\hline $\begin{array}{l}\text { Age (year), mean } \pm \mathrm{SD} \\
\text { (range) }\end{array}$ & $\begin{array}{l}29.0 \pm 5.3 \\
(17-39)\end{array}$ & $\begin{array}{l}28.9 \pm 5.2 \\
(17-39)\end{array}$ & $\begin{array}{l}27.6 \pm 5.0 \\
(17-37)\end{array}$ & $\begin{array}{l}30.2 \pm 5.2 \\
(22-39)\end{array}$ \\
\hline \multicolumn{5}{|l|}{ Gender, n (\%) } \\
\hline Male & $17(37)$ & $17(40)$ & $9(41)$ & $8(40)$ \\
\hline Female & $29(63)$ & $25(60)$ & $13(59)$ & $12(60)$ \\
\hline \multicolumn{5}{|l|}{ Race, n (\%) } \\
\hline $\begin{array}{l}\text { American Indian or Alaska Native, } \\
\text { White }\end{array}$ & $1(2)$ & $1(2)$ & $1(5)$ & $0(0)$ \\
\hline Asian & $1(2)$ & $1(2)$ & $1(5)$ & $0(0)$ \\
\hline Asian, White & $1(2 \%)$ & $1(2)$ & $1(5)$ & $0(0)$ \\
\hline Black or African-American & $4(9)$ & $4(10 \%$ & $4(18)$ & $0(0)$ \\
\hline White & $39(85)$ & $35(83)$ & $15(68)$ & $20(100)$ \\
\hline \multicolumn{5}{|l|}{ Ethnicity, n (\%) } \\
\hline Hispanic or Latino & $0(0)$ & $0(0)$ & $0(0)$ & $0(0)$ \\
\hline Not Hispanic or Latino & 46 (100) & $42(100)$ & $22(100)$ & $20(100)$ \\
\hline \multicolumn{5}{|l|}{ Education, n (\%) } \\
\hline 9th-11th grade & $1(2)$ & $1(2)$ & $1(5)$ & $0(0)$ \\
\hline 12th grade & $1(2)$ & $1(2)$ & $0(0)$ & $1(5)$ \\
\hline Some college/associate degree & $5(11)$ & $3(7)$ & $3(14)$ & $0(0)$ \\
\hline College degree & $26(57)$ & $25(60)$ & $13(59)$ & $12(60)$ \\
\hline Non-doctoral graduate degree & $9(20)$ & $8(19)$ & $4(18)$ & $4(20)$ \\
\hline Doctoral degree & $4(9)$ & $4(10)$ & $1(5)$ & $3(15)$ \\
\hline \multicolumn{5}{|l|}{ Study limb, n (\%) } \\
\hline Left & $21(46)$ & $19(45)$ & $10(45)$ & $9(45)$ \\
\hline Right & $25(54)$ & $23(55)$ & $12(55)$ & $11(55)$ \\
\hline \multicolumn{5}{|l|}{ Pain: involved side, n (\%) } \\
\hline Left & $15(33)$ & $13(31)$ & $8(36)$ & $5(25)$ \\
\hline Right & $15(33)$ & $13(31)$ & $6(27)$ & $7(35)$ \\
\hline Bilateral & $16(35)$ & $16(38)$ & $8(36)$ & $8(40)$ \\
\hline $\begin{array}{l}\text { Measured BMI }\left(\mathrm{kg} / \mathrm{m}^{2}\right) \text {, mean } \pm S D \\
\text { (range) }\end{array}$ & $\begin{array}{l}25.6 \pm 6.3 \\
(17.9-46.1)\end{array}$ & $\begin{array}{l}25.2 \pm 6.0 \\
(17.9-46.1)\end{array}$ & $\begin{array}{l}24.9 \pm 6.1 \\
(17.9-41.8)\end{array}$ & $\begin{array}{l}25.6 \pm 6.1 \\
(20.2-46.1)\end{array}$ \\
\hline UCLA*, median (range) & $10(3-10)$ & $10(3-10)$ & $9.5(3-10)$ & $10(4-10)$ \\
\hline \multicolumn{5}{|l|}{$\begin{array}{l}\text { Pain report, } \\
\text { median } \\
\text { (range) }\end{array}$} \\
\hline Pain duration (year) & $\begin{array}{l}2.0 \\
(0.3-17.0)\end{array}$ & $\begin{array}{l}2.0 \\
(0.3-17.0)\end{array}$ & $\begin{array}{l}1.8 \\
(0.3-12.0)\end{array}$ & $\begin{array}{l}3.0 \\
(0.3-17.0)\end{array}$ \\
\hline Average pain $†$ & $3(1-9)$ & $3(1-9)$ & $4(1-9)$ & $3(1-8)$ \\
\hline Worst pain† & $6(2-10)$ & $6(2-10)$ & $6(2-9)$ & $5.5(3-10)$ \\
\hline
\end{tabular}

*Patients are asked to rate their activity level over the previous 6 months. $10=$ regularly participates in impact sports; $1=$ wholly inactive, dependent on others.

†Pain rated by patients using a verbal numerical pain rating scale. $0=$ no pain; $10=$ worst pain imaginable.

BMI, body mass index; MoveTrain, movement pattern training group; UCLA, University of California Los Angeles Activity Score. 
Table 2 Summary of results for primary outcomes related to study feasibility

\begin{tabular}{|c|c|c|c|c|c|c|c|}
\hline \multirow[b]{2}{*}{ Variable } & \multirow[b]{2}{*}{$\begin{array}{l}\text { All } \\
n=46\end{array}$} & \multicolumn{3}{|c|}{ Patients, by site } & \multicolumn{3}{|c|}{ Patients, by treatment group } \\
\hline & & $\begin{array}{l}\text { Primary } \\
\mathrm{n}=30\end{array}$ & $\begin{array}{l}\text { Partner } \\
n=16\end{array}$ & $\mathbf{P}$ & $\begin{array}{l}\text { MoveTrain } \\
\mathrm{n}=23\end{array}$ & $\begin{array}{l}\text { Standard } \\
n=23\end{array}$ & $\mathbf{P}$ \\
\hline Retention rate, $\mathrm{n}(\%)^{*}$ & $42(91)$ & $30(100)$ & $12(75)$ & $0.01 \dagger$ & $22(96)$ & $20(87)$ & $0.61 \dagger$ \\
\hline $\begin{array}{l}\text { Patient adherent to treatment } \\
\text { session attendance, } \mathrm{n}(\%) \ddagger\end{array}$ & $\begin{array}{l}\text { All } \\
n=43 \S\end{array}$ & $\begin{array}{l}\text { Primary } \\
n=30 \S\end{array}$ & $\begin{array}{l}\text { Partner } \\
n=13 \S\end{array}$ & $\mathbf{P}$ & $\begin{array}{l}\text { MoveTrain } \\
\mathrm{n}=21 \S\end{array}$ & $\begin{array}{l}\text { Standard } \\
\mathrm{n}=22 \S\end{array}$ & $\mathbf{P}$ \\
\hline \multirow{2}{*}{$\begin{array}{l}\text { Patient treatment receipt, } \\
\text { median \% } \\
\text { (range)†† }\end{array}$} & $\begin{array}{l}91 \\
(18-100)\end{array}$ & $\begin{array}{l}92 \\
(68-100)\end{array}$ & $\begin{array}{l}83 \\
(18-98)\end{array}$ & $0.47^{\star \star}$ & $\begin{array}{l}88 \\
(18-100)\end{array}$ & $\begin{array}{l}93 \\
(81-100)\end{array}$ & $0.05^{\star \star}$ \\
\hline & $\begin{array}{l}\text { All } \\
n=45 \neq \ddagger\end{array}$ & $\begin{array}{l}\text { Primary } \\
\mathrm{n}=30 \neq \pm\end{array}$ & $\begin{array}{l}\text { Partner } \\
\mathrm{n}=15 \neq \ddagger\end{array}$ & $\mathbf{P}$ & $\begin{array}{l}\text { MoveTrain } \\
\mathrm{n}=30 \neq \neq\end{array}$ & $\begin{array}{l}\text { Standard } \\
n=15 \neq \ddagger\end{array}$ & $\mathbf{P}$ \\
\hline
\end{tabular}

${ }^{*}$ Retention rate is defined as the percentage of those enrolled at baseline who completed testing after treatment.

†Fisher's exact test for group comparison.

¥Treatment session attendance was documented by the physical therapist. The variable for treatment session attendance is the number of patients who attended at least $90 \%$ (9 of 10$)$ of their sessions.

$\S$ Number of patients completing at least nine supervised visits.

IIHEP adherence: participants were instructed to perform their HEP one time per day. The variable for HEP adherence was calculated as the total number of days reported by the participant to have completed the prescribed exercises, summed over the entire treatment period and divided by the total number of treatment days.

**Wilcoxon's test.

††The total number of exercises the physical therapist reviewed and the total number of those exercises they rated as independent were each summed across all treatment visits to derive the per cent of exercises rated as independent across the entire treatment $\left(\Sigma\right.$ independent / $\Sigma$ reviewed $\left.{ }^{*} 100\right)$.

$\ddagger \ddagger N u m b e r$ of patients who completed at least one supervised treatment visit.

$\S \S$ Treatment delivery assessed using chart reviews to determine if the active ingredients of the treatment was provided. The variable for treatment delivery is the proportion of active ingredients completed and documented by the treatment physical therapists for 132 treatment visits.

HEP, home exercise programme; MoveTrain, movement pattern training group.

increases in strength after treatment ranging from $6 \%$ to $16 \%$ (table 5 ).

\section{DISCUSSION}

We have established feasibility of completing a large, multicentre RCT to compare effectiveness of MoveTrain to Standard. We met our target enrolment and retention rate. Patient adherence to treatment session attendance was high. however, patient-reported adherence to HEP was lower than expected. Regarding treatment fidelity, we demonstrated high rates of treatment delivery among PTs and treatment receipt among patients. Our preliminary results related to secondary outcomes suggest both

Table 3 The number of patients who reported pain or discomfort with at least one exercise during treatment

\begin{tabular}{lccc}
\hline Variable $^{*}$ & MoveTrain & Standard & Total \\
\hline Hip & 7 & 13 & 20 \\
Knee & & 1 & 1 \\
Lumbar spine & 1 & & 1 \\
Hip and lumbar spine & 1 & & 1 \\
Hip and knee & 1 & 3 & 4 \\
Total & 10 & 17 & 27 \\
\hline
\end{tabular}

Exercises were modified by the treating physical therapist.

*Each number represents one participant.

MoveTrain, movement pattern training group.
MoveTrain and Standard resulted in clinically significant improvements in patient-reported outcomes and hip muscle strength; however, only those in MoveTrain showed improvement in kinematics.

\section{Feasibility}

We were able to meet our recruitment goals within 13 months of initiating recruitment. This suggests our recruitment methods would result in an estimated 177 patients for a 5-year project. Our retention rate for post-treatment testing was $91 \%$, higher than the $80 \%$ follow-up required for a high-quality RCT. ${ }^{31}$ We provided compensation for testing sessions and treatment free of charge, which may have increased retention. Additionally, there was frequent contact between study personnel and patients, including weekly treatment sessions and multiple communications with research coordinators.

Treatment session attendance was high, indicating feasibility of weekly supervised sessions. Patient selfreported HEP adherence was lower than hypothesised. Based on informal discussions and written communications, patients reported difficulty completing all exercises every day and suggested the HEP be modified. Despite lower than expected HEP adherence, patients in both groups demonstrated improvements in HOOS and treatment-targeted mechanism, kinematics and strength. Decreasing HEP frequency may result in 
Table 4 Summary of results for patient-reported outcome measures $(n=42)$

\begin{tabular}{|c|c|c|c|c|c|}
\hline Variable & $\begin{array}{l}\text { Pretest } \\
\text { Mean } \pm \text { SD }\end{array}$ & $\begin{array}{l}\text { Post-test } \\
\text { Mean } \pm S D\end{array}$ & $\begin{array}{l}\text { Within-group } \\
\text { change }^{*} \\
\text { Mean } \pm \text { SD }\end{array}$ & $\begin{array}{l}\text { Adjusted immediate } \\
\text { treatment effect† } \\
\text { Mean }(95 \% \mathrm{Cl})\end{array}$ & $P$ value $\neq$ \\
\hline \multicolumn{6}{|l|}{ HOOS scores } \\
\hline \multicolumn{6}{|l|}{ HOOSPain§ } \\
\hline MoveTrain $(n=22)$ & $66.6 \pm 14.9$ & $84.7 \pm 13.5$ & $18.1 \pm 12.9$ & $-2.75(-8.66$ to 3.16$)$ & 0.35 \\
\hline Standard $(n=20)$ & $70.3 \pm 12.0$ & $89.3 \pm 8.7$ & $19.0 \pm 9.6$ & & \\
\hline \multicolumn{6}{|l|}{ HOOSSymptoms§ } \\
\hline MoveTrain $(n=22)$ & $63.2 \pm 16.6$ & $78.0 \pm 14.1$ & $15.0 \pm 13.2$ & $-5.55(-13.10$ to 1.99$)$ & 0.14 \\
\hline Standard $(n=20)$ & $68.8 \pm 15.5$ & $86.3 \pm 13.5$ & $17.5 \pm 16.2$ & & \\
\hline \multicolumn{6}{|l|}{ HOOSADL§ } \\
\hline MoveTrain $(n=22)$ & $79.5 \pm 15.0$ & $92.0 \pm 11.2$ & $12.5 \pm 11.0$ & $-2.20(-6.51$ to 2.11$)$ & $0.32^{A}$ \\
\hline Standard $(n=20)$ & $83.4 \pm 14.7$ & $95.7 \pm 6.2$ & $12.4 \pm 11.8$ & & \\
\hline \multicolumn{6}{|l|}{ HOOSSport§ } \\
\hline MoveTrain $(n=22)$ & $58.5 \pm 20.0$ & $83.0 \pm 16.4$ & $24.4 \pm 18.1$ & $-3.69(-11.74$ to 4.36$)$ & 0.36 \\
\hline Standard $(n=20)$ & $69.1 \pm 17.6$ & $90.6 \pm 11.2$ & $21.6 \pm 15.6$ & & \\
\hline \multicolumn{6}{|l|}{ HOOSQOL§ } \\
\hline MoveTrain $(n=22)$ & $55.1 \pm 14.8$ & $68.2 \pm 16.8$ & $13.1 \pm 13.8$ & $-6.60(-15.32$ to 2.13$)$ & 0.13 \\
\hline Standard $(n=20)$ & $52.2 \pm 15.2$ & $73.1 \pm 15.3$ & $20.9 \pm 16.6$ & & \\
\hline \multicolumn{6}{|c|}{ PSFS, mean of up to five activities } \\
\hline \multicolumn{6}{|l|}{ PSFSף } \\
\hline MoveTrain $(n=22)$ & $5.8 \pm 1.9$ & $7.5 \pm 2.2$ & $1.7 \pm 1.7$ & $-0.09(-1.20$ to 1.02$)$ & 0.87 \\
\hline Standard $(n=20)$ & $5.3 \pm 1.5$ & $7.2 \pm 2.2$ & $1.8 \pm 1.8$ & & \\
\hline
\end{tabular}

PROMIS T-scores ${ }^{* *}$

PROMIS mobility

\begin{tabular}{|c|c|c|c|c|c|}
\hline MoveTrain $(n=22)$ & $50.0 \pm 5.7$ & $53.5 \pm 6.1$ & $3.5 \pm 4.8$ & $-2.05(-5.11$ to 1.01$)$ & 0.18 \\
\hline Standard $(n=20)$ & $47.9 \pm 6.2$ & $54.3 \pm 5.9$ & $6.4 \pm 5.8$ & & \\
\hline \multicolumn{6}{|c|}{ PROMIS pain interference } \\
\hline MoveTrain $(n=22)$ & $53.6 \pm 5.0$ & $46.8 \pm 7.8$ & $-6.8 \pm 6.3$ & \multirow[t]{2}{*}{-0.51 ( -4.31 to 3.29$)$} & \multirow[t]{2}{*}{$0.79 \dagger \dagger$} \\
\hline Standard $(n=20)$ & $54.6 \pm 4.3$ & $48.1 \pm 6.7$ & $-6.5 \pm 6.6$ & & \\
\hline \multicolumn{6}{|c|}{ PROMIS pain behaviour } \\
\hline MoveTrain $(n=22)$ & $55.1 \pm 3.2$ & $46.6 \pm 9.0$ & $-8.4 \pm 7.5$ & \multirow[t]{2}{*}{$-0.47(-5.24$ to 4.30$)$} & \multirow[t]{2}{*}{$0.85 † \dagger$} \\
\hline Standard $(n=20)$ & $55.2 \pm 2.7$ & $47.3 \pm 8.4$ & $-7.9 \pm 8.5$ & & \\
\hline \multicolumn{6}{|c|}{ PROMIS global physical health } \\
\hline MoveTrain $(n=22)$ & $49.7 \pm 6.1$ & $56.1 \pm 7.4$ & $6.4 \pm 5.4$ & \multirow[t]{2}{*}{$0.11(-3.07$ to 3.29$)$} & \multirow[t]{2}{*}{$0.95 \dagger \dagger$} \\
\hline Standard $(n=20)$ & $49.9 \pm 5.7$ & $56.2 \pm 6.4$ & $6.2 \pm 5.7$ & & \\
\hline \multicolumn{6}{|c|}{ PROMIS global mental health } \\
\hline MoveTrain $(n=22)$ & $52.3 \pm 9.4$ & $53.8 \pm 11.2$ & $1.5 \pm 5.6$ & \multirow[t]{2}{*}{$-1.82(-5.47$ to 1.83$)$} & \multirow[t]{2}{*}{0.32} \\
\hline Standard $(n=20)$ & $55.5 \pm 7.9$ & $58.7 \pm 8.5$ & $3.2 \pm 5.7$ & & \\
\hline \multicolumn{6}{|c|}{ Pain numeric rating scales } \\
\hline \multicolumn{6}{|l|}{ Pain (average) $\ddagger \ddagger$} \\
\hline MoveTrain $(n=22)$ & $4.1 \pm 2.0$ & $1.3 \pm 1.5$ & $-2.9 \pm 1.5$ & \multirow[t]{2}{*}{$0.21(-0.96$ to 1.38$)$} & \multirow[t]{2}{*}{$0.73 \S \S$} \\
\hline Standard $(n=20)$ & $3.5 \pm 1.5$ & $1.0 \pm 1.4$ & $-2.5 \pm 1.9$ & & \\
\hline
\end{tabular}




\begin{tabular}{|c|c|c|c|c|c|}
\hline Variable & $\begin{array}{l}\text { Pretest } \\
\text { Mean } \pm \text { SD }\end{array}$ & $\begin{array}{l}\text { Post-test } \\
\text { Mean } \pm \text { SD }\end{array}$ & $\begin{array}{l}\text { Within-group } \\
\text { change* } \\
\text { Mean } \pm \text { SD }\end{array}$ & $\begin{array}{l}\text { Adjusted immediate } \\
\text { treatment effect } \\
\text { Mean }(95 \% \mathrm{Cl})\end{array}$ & P value $\ddagger$ \\
\hline \multicolumn{6}{|l|}{ 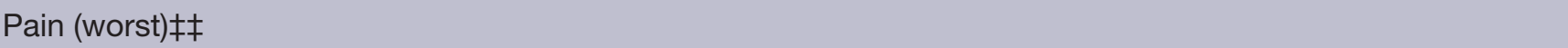 } \\
\hline MoveTrain (n=22) & $5.6 \pm 2.0$ & $2.0 \pm 1.7$ & $-3.7 \pm 1.8$ & $0.36(-0.74$ to 1.46$)$ & $0.52 \S \S$ \\
\hline Standard $(n=20)$ & $5.8 \pm 1.9$ & $1.9 \pm 2.2$ & $-3.9 \pm 3.0$ & & \\
\hline
\end{tabular}

${ }^{*}$ Change is calculated by subtracting the pretest value from the post-test value.

†The adjusted treatment effect from least squares means is calculated by subtracting the MoveTrain minus Standard at post-test and assesses the between-group difference in post-test values after adjusting for pretest.

łUnless otherwise indicated, $\mathrm{p}$ value by analysis of covariance using a generalised linear model.

§Patient-reported outcome measures with 100=no disability.

IPatient Specific Functional Scale (PSFS) assesses the level of difficulty performing up to five activities due to hip pain or symptoms. $0=$ unable to perform activity; $10=$ able to perform activity at preinjury level.

${ }^{*}$ PROMIS T-scores for the measures included in this study range from 20 to 80 and have a mean score of 50 and SD of 10, using the US general population as the reference. A higher score represents more of the concept being measured. Minimally important differences (MIDs) have not been established for those with HRGP. More information regarding PROMIS score interpretation may be found the following website: http://www.healthmeasures.net/score-and-interpret/interpret-scores/promis

$\dagger † P$ value by non-parametric analysis of covariance.

$\ddagger \ddagger$ Pain rated by patients using a verbal numerical pain rating scale. $0=$ no pain; $10=$ worst pain imaginable. $\S \S \mathrm{P}$ value by analysis of covariance using multinomial generalised estimating equations with a cumulative logit.

HOOS, Hip Disability and Osteoarthritis Outcome Score; HOOSADL, function in activities of daily living; HOOSQOL, quality of life; HOOSSport, function in sports and recreation; HRGP, hip-related groin pain; MoveTrain, movement pattern training group; PROMIS, Patient-Reported Outcomes Measurement Information System.

similar treatment effects and assist in improving HEP adherence.

We have developed methods to establish and monitor treatment fidelity. In the future, we will use the checklist used for role-played scenarios to monitor actual treatment sessions. For chart reviews, we noted the two items most often scored as insufficient included 'review of key concepts' and 'addressing barriers to HEP completion'. Discussion with treating PTs indicated they indeed performed these tasks; however, they did not specifically document their strategies. In the future, we will modify treating PTs' documentation forms to provide a specific place to document these two key items. This addition will serve as an improved method to ensure documentation of protocol adherence and as a reminder to treating PTs to perform these items.

\section{Preliminary effectiveness}

Our study was designed to assess feasibility; therefore, we did not expect to find statistical differences in secondary outcome measures. Both groups demonstrated similar improvements in all patient-reported outcomes. These findings are important because it suggests that patients with HRGP may benefit from PT-led intervention. Patients in this study reported moderate activity limitations at baseline. After treatment, HOOS subscales were substantially improved with increases of $12-24$ points. No unexpected adverse events occurred, suggesting these interventions are safe.

After treatment, MoveTrain did demonstrate a significantly larger reduction in hip adduction and pelvic drop during single leg squat compared with Standard, supporting our a priori hypothesis. The goal of MoveTrain training is to improve lower extremity kinematics during daily and patient-specific tasks. Because MoveTrain changes the way people move, stresses on joint tissues may be altered, potentially resulting in greater long-term effects than Standard. Interestingly, fewer patients in MoveTrain reported discomfort with their exercises (table 3 ). We are currently collecting patientreported outcome measures 6 and 12 months after treatment to determine if between-group differences emerge over time. Further investigation is warranted to determine if the improved movement patterns can be sustained over time and have a protective influence on the hip.

There were similar strength improvements, ranging from $6 \%$ to $16 \%$, in both patient groups. Although the MoveTrain group did not perform traditional strengthening exercises, the functional tasks performed MoveTrain incorporated general principles of strength 
Table 5 Summary of results for preliminary effectiveness variable (targeted impairments) $(n=42)$

\begin{tabular}{|c|c|c|c|c|}
\hline Variable & $\begin{array}{l}\text { Pretest } \\
\text { Mean } \pm \text { SD }\end{array}$ & $\begin{array}{l}\text { Post-test } \\
\text { Mean } \pm \text { SD }\end{array}$ & $\begin{array}{l}\text { Within-group change* } \\
\text { Mean } \pm \text { SD }\end{array}$ & $\begin{array}{l}\text { Adjusted immediate } \\
\text { treatment effect† } \\
\text { Mean }(95 \% \mathrm{Cl})\end{array}$ \\
\hline
\end{tabular}

Hip kinematics for the study side $\left({ }^{\circ}\right) \S$

Hip adduction angleף

$\begin{array}{llccc}\text { MoveTrain }(n=22) & 23.5 \pm 11.4 & 15.6 \pm 9.5 & -7.9 \pm 12.5 & -7.2(-12.9 \text { to }-1.4) \\ \text { Standard }(n=20) & 16.9 \pm 11.0 & 19.4 \pm 11.3 & 2.5 \pm 7.1 & 016\end{array}$

Pelvic tilt excursion**

\begin{tabular}{|c|c|c|c|c|c|}
\hline MoveTrain $(n=22)$ & $-10.7 \pm 5.1$ & $-7.5 \pm 4.6$ & $3.3 \pm 5.7$ & 3.0 (0.4 to 5.6$)$ & 0.026 \\
\hline Standard $(n=20)$ & $-8.6 \pm 7.2$ & $-9.5 \pm 5.4$ & $-0.8 \pm 4.6$ & & \\
\hline \multicolumn{6}{|c|}{ Trunk lean excursion ${ }^{\star \star}$} \\
\hline MoveTrain $(n=22)$ & $3.8 \pm 6.6$ & $2.8 \pm 3.9$ & $-1.0 \pm 6.3$ & $-1.1(-3.6$ to 1.4$)$ & 0.37 \\
\hline Standard $(n=20)$ & $7.5 \pm 8.9$ & $5.3 \pm 5.6$ & $-2.2 \pm 6.3$ & & \\
\hline
\end{tabular}

Hip muscle strength for the study side††

\begin{tabular}{|c|c|c|c|c|c|}
\hline \multicolumn{6}{|l|}{ ABDs, torque $(\mathrm{Nm})$} \\
\hline MoveTrain (n=22) & $7.3 \pm 3.3$ & $8.4 \pm 3.1$ & $1.2 \pm 2.1$ & \multirow[t]{2}{*}{$0.3(-0.9$ to 1.5$)$} & \multirow[t]{2}{*}{0.65} \\
\hline Standard $(n=20)$ & $6.8 \pm 3.6$ & $7.8 \pm 2.9$ & $1.0 \pm 2.3$ & & \\
\hline \multicolumn{6}{|l|}{ ERs $90^{\circ}$ torque $(\mathrm{Nm})$} \\
\hline MoveTrain $(n=22)$ & $2.3 \pm 1.0$ & $2.5 \pm 0.9$ & $0.2 \pm 0.8$ & \multirow[t]{2}{*}{$-0.2(-0.6$ to 0.3$)$} & \multirow[t]{2}{*}{0.44} \\
\hline Standard $(n=20)$ & $2.3 \pm 1.1$ & $2.7 \pm 1.1$ & $0.4 \pm 0.7$ & & \\
\hline \multicolumn{6}{|l|}{ IRs $90^{\circ}$ torque $(\mathrm{Nm})$} \\
\hline MoveTrain $(n=22)$ & $2.5 \pm 0.8$ & $2.8 \pm 0.8$ & $0.2 \pm 0.7$ & \multirow[t]{2}{*}{$-0.1(-0.6$ to 0.5$)$} & \multirow[t]{2}{*}{0.73} \\
\hline Standard $(n=20)$ & $2.3 \pm 0.9$ & $2.7 \pm 1.2$ & $0.4 \pm 1.1$ & & \\
\hline \multicolumn{6}{|l|}{ ADDs, torque $(\mathrm{Nm})$} \\
\hline MoveTrain ( $n=21)$ & $4.4 \pm 2.0$ & $5.3 \pm 2.4$ & $0.9 \pm 1.3$ & \multirow[t]{2}{*}{$0.2(-0.6$ to 1.1$)$} & \multirow[t]{2}{*}{$0.60 \neq \ddagger$} \\
\hline Standard $(n=20)$ & $4.7 \pm 3.1$ & $5.2 \pm 2.1$ & $0.5 \pm 1.9$ & & \\
\hline \multicolumn{6}{|l|}{ Flexs, torque $(\mathrm{Nm})$} \\
\hline MoveTrain $(n=22)$ & $5.8 \pm 2.1$ & $7.0 \pm 2.6$ & $1.3 \pm 2.1$ & \multirow[t]{2}{*}{$0.6(-0.6$ to 1.7$)$} & \multirow[t]{2}{*}{0.34} \\
\hline Standard $(n=19)$ & $5.5 \pm 1.8$ & $6.3 \pm 1.9$ & $0.8 \pm 1.6$ & & \\
\hline \multicolumn{6}{|l|}{ Exts, torque (Nm) } \\
\hline MoveTrain $(n=22)$ & $8.8 \pm 4.0$ & $9.4 \pm 4.0$ & $0.5 \pm 2.7$ & \multirow[t]{2}{*}{$-0.5(-2.1$ to 1.0$)$} & \multirow[t]{2}{*}{0.51} \\
\hline Standard $(n=19)$ & $8.9 \pm 4.4$ & $9.9 \pm 4.4$ & $1.0 \pm 2.3$ & & \\
\hline
\end{tabular}

${ }^{*}$ Change is calculated by subtracting the pretest value from the post-test value.

†The adjusted treatment effect from least squares mean is calculated by subtracting the MoveTrain minus Standard at post-test, and assesses the between-group difference in post-test values after adjusting for pretest.

łUnless otherwise indicated, $p$ value by analysis of covariance using a generalised linear model.

§Kinematics represent the mean of up to three trials where the sign of the measurements (ie, negative or positive) are included when computing the average.

ПHip adduction angle was measures at the lowest depth of the single leg squat. Larger positive values indicate a larger hip adduction angle.

${ }^{* *}$ Values for excursion were calculated from first initiation of descent to the end of descent on the stance leg. Pelvic tilt excursion: larger negative values indicate a larger pelvic drop. Trunk lean: larger positive values indicate trunk lean towards the weightbearing limb.

$\dagger †$ Muscle torque $(\mathrm{Nm})$ was normalised by body weight $(\mathrm{N}) \times$ height $(\mathrm{m}) \times 100$ using the mean of up to three trials. 抽 value by non-parametric analysis of covariance.

ABDs, abductors with the hip in $0^{\circ}$ hip flexion, $0^{\circ}$ abduction and $0^{\circ}$ rotation; ADDs, adductors with the hip in $0^{\circ}$ hip flexion, $0^{\circ}$ abduction and $0^{\circ}$ rotation; ERs $90^{\circ}$, external rotator strength with hip in $90^{\circ}$ flexion, $0^{\circ}$ abduction and $0^{\circ}$ rotation; Exts, extensors with the hip in $0^{\circ}$ hip flexion, $0^{\circ}$ abduction and $0^{\circ}$ rotation; Flexs, flexors with the hip in $0^{\circ}$ hip flexion, $0^{\circ}$ abduction and $0^{\circ}$ rotation; IRs $90^{\circ}$, internal rotator strength with hip in $90^{\circ}$ flexion, $0^{\circ}$ abduction and $0^{\circ}$ rotation; MoveTrain, movement pattern training group.

training. Based on patient performance, the tasks were progressed by level of difficulty or by increasing resistance. Therefore, these progressive activities may have contributed to improved muscle strength.

\section{Limitations}

We cannot make definitive statements regarding efficacy of MoveTrain or Standard treatment; however, our results are promising. Improvements were reported in both 
treatment groups, and those in MoveTrain improved their kinematics. We did not have a control group; therefore, patient improvement may be related to passage of time. We consider this unlikely, because in our proofof-concept study, ${ }^{9}$ patients with HRGP randomised to a 6-week waitlist demonstrated a worsening in HOOSADL and no improvement in other HOOS subscales compared with baseline. Additionally, our patients reported pain duration greater than 3 months prior to enrolling, suggesting that time alone may not explain our results. We must acknowledge that we have completed a number of statistical tests, therefore increasing the likelihood of a type I error. We identified our a priori variables of interest and focused our discussion on results related to these variables. However, given limited information related to non-operative management of HRGP, we believe it important to report all analyses that may be useful for future investigations. Finally, we did not establish test-retest reliability of the strength measures used in this pilot study; however, we will include reliability testing for the future trial.

We completed a pilot, multicentre RCT to assess our capability of completing a larger, definitive RCT to assess the efficacy of MoveTrain. We encountered no major problems and have determined a full study can proceed with modifications. Preliminary results from this study suggests that both MoveTrain and Standard may result in improved patient-reported outcomes; however, only MoveTrain resulted in improved lower extremity kinematics. Additionally, fewer patients reported discomfort with the exercises provided in MoveTrain, which may influence patient adherence to treatment. Further investigation of MoveTrain is warranted. We do not know if the improvements in patient-reported outcomes observed in this study were due to the treatment provided or due to the fact that the participants interacted with a healthcare professional. An adequately powered RCT is needed to assess the efficacy of MoveTrain compared with an attention control. Future work will also include the assessment of the sustained benefits of PT-led interventions.

\author{
Author affiliations \\ ${ }^{1}$ Department of Physical Therapy, Washington University School of Medicine in Saint \\ Louis, Saint Louis, Missouri, USA \\ ${ }^{2}$ Department of Orthopaedic Surgery, Washington University School of Medicine, St. \\ Louis, Missouri, USA \\ ${ }^{3}$ Division of Biostatistics, Washington University in Saint Louis, Saint Louis, \\ Missouri, USA \\ ${ }^{4}$ Department of Physical Therapy, University of Pittsburgh, Pittsburgh, Pennsylvania, \\ USA \\ ${ }^{5}$ Department of Physical Therapy, Washington University in Saint Louis, Saint Louis, \\ Missouri, USA \\ ${ }^{6}$ Department of Orthopaedic Surgery, Washington University in Saint Louis, Saint \\ Louis, Missouri, USA
}

Twitter Marcie Harris-Hayes @MHarrisHayes

Acknowledgements The authors would like to acknowledge Megan Burgess, Visnja King, Suzanne Kuebler, Joseph Mancino and Dave Wortman for providing treatment during the trial; Kathy Brown, Martha Hessler and Darrah Snozek their assistance with trial procedures and data collection.

Contributors All authors contributed to the study design, data analysis, interpretation and manuscript preparation, including final approval. The corresponding author takes responsibility for the integrity of the work as a whole, from inception to the finished article. $\mathrm{MH}-\mathrm{H}$ : conception and design of study, data analysis and interpretation; drafting, revising and approving the final manuscript; provision of study materials and patients; obtaining of funding; and collection and assembly of data. KS-M: conception and design of study, data analysis and interpretation; drafting, revising and approving the final manuscript; statistical expertise; administrative, technical or logistic support; and collection and assembly of data. AMB: conception and design of study, data analysis and interpretation; drafting, revising and approving the final manuscript; provision of study materials and patients; administrative, technical or logistic support; and collection and assembly of data. SNF: data analysis and interpretation; drafting, revising and approving the final manuscript; and collection and assembly of data. MJM and JCC: conception and design of study, data analysis and interpretation; drafting, revising and approving the final manuscript; and provision of study materials and patients. GKF: conception and design of study, data analysis and interpretation; drafting, revising and approving the final manuscript; provision of study materials and patients; obtaining of funding; and collection and assembly of data.

Funding This work was supported by the following grants: R21HD086644 and NIH T32HD007434 from the National Center for Medical Rehabilitation Research, National Institute of Child Health and Human Development; the Orthopaedic Research Grant from the Foundation for Physical Therapy Research; Washington University Institute of Clinical and Translational Sciences grant UL1TR002345 from the National Center for Advancing Translational Sciences of the National Institutes of Health. Additional support was provided by Program in Physical Therapy at Washington University School of Medicine, Clinical and Translational Science Award.

\section{Competing interests None declared.}

Patient and public involvement Patients and/or the public were not involved in the design, or conduct, or reporting, or dissemination plans of this research.

Patient consent for publication Not required.

Provenance and peer review Not commissioned; externally peer reviewed.

Data availability statement Data are available on reasonable request. Deidentified participant data related to this study may be made available on reasonable request to the principal investigator, MHH. Email: harrisma@wustl.edu.

Open access This is an open access article distributed in accordance with the Creative Commons Attribution Non Commercial (CC BY-NC 4.0) license, which permits others to distribute, remix, adapt, build upon this work non-commercially, and license their derivative works on different terms, provided the original work is properly cited, appropriate credit is given, any changes made indicated, and the use is non-commercial. See: http://creativecommons.org/licenses/by-nc/4.0/.

ORCID iD

Marcie Harris-Hayes http://orcid.org/0000-0003-4274-1651

\section{REFERENCES}

1 Agricola R, Heijboer MP, Bierma-Zeinstra SMA, et al. Cam impingement causes osteoarthritis of the hip: a nationwide prospective cohort study (check). Ann Rheum Dis 2013;72:918-23.

2 Harris-Hayes M, Royer NK. Relationship of acetabular dysplasia and femoroacetabular impingement to hip osteoarthritis: a focused review. Pm $R$ 2011;3:1055-67.

3 Ayeni OR, Belzile EL, Musahl V, et al. Results of the perception of femoroacetabular impingEment by surgeons survey (process). Knee Surg Sports Traumatol Arthrosc 2014;22:906-10.

$4 \mathrm{Ng}$ VY, Arora N, Best TM, et al. Efficacy of surgery for femoroacetabular impingement: a systematic review. Am J Sports Med 2010;38:2337-45.

5 Wall PDH, Fernandez M, Griffin DR, et al. Nonoperative treatment for femoroacetabular impingement: a systematic review of the literature. Pm R 2013;5:418-26.

6 Griffin DR, Dickenson EJ, Wall PDH, et al. Hip arthroscopy versus best conservative care for the treatment of femoroacetabular impingement syndrome (UK fashion): a multicentre randomised controlled trial. Lancet 2018;391:2225-35.

7 Palmer AJR, Ayyar Gupta V, Fernquest S, et al. Arthroscopic hip surgery compared with physiotherapy and activity modification 
for the treatment of symptomatic femoroacetabular impingement: multicentre randomised controlled trial. BMJ 2019;364:1185.

8 Mansell NS, Rhon DI, Meyer J, et al. Arthroscopic surgery or physical therapy for patients with femoroacetabular impingement syndrome: a randomized controlled trial with 2-year follow-up. Am J Sports Med 2018;46:1306-14.

9 Harris-Hayes M, Czuppon S, Van Dillen LR, et al. Movement-pattern training to improve function in people with chronic hip joint pain: a feasibility randomized clinical trial. J Orthop Sports Phys Ther 2016:46:452-61.

10 Harris-Hayes M, Steger-May K, Van Dillen LR, et al. Reduced hip adduction is associated with improved function after movementpattern training in young people with chronic hip joint pain. $J$ Orthop Sports Phys Ther 2018:1-28.

11 Borrelli B. The assessment, monitoring, and enhancement of treatment fidelity in public health clinical trials. J Public Health Dent 2011;71:S52-63.

12 Borrelli B, Sepinwall D, Ernst D, et al. A new tool to assess treatment fidelity and evaluation of treatment fidelity across 10 years of health behavior research. J Consult Clin Psychol 2005;73:852-60.

13 MacDonald SJ, Garbuz D, Ganz R. Clinical examination of the symptomatic young adult hip. Sem Arthroplasty 1997;8:3-9.

14 Byrd JW, Jones KS. Prospective analysis of hip arthroscopy with 2-year follow-up. Arthroscopy 2000;16:578-87.

15 Hoffmann TC, Glasziou PP, Boutron I, et al. Better reporting of interventions: template for intervention description and replication (TIDieR) checklist and guide. BMJ 2014;348:g1687.

16 Stratford P, Gill C, Westaway M. Assessing disability and change on individual patients: a report of a patient specific measure. Physiotherapy Canada 1995;47:258-63.

17 Enseki K, Harris-Hayes M, White DM, et al. Nonarthritic hip joint pain. J Orthop Sports Phys Ther 2014;44:A1-32.

18 Bennell KL, O'Donnell JM, Takla A, et al. Efficacy of a physiotherapy rehabilitation program for individuals undergoing arthroscopic management of femoroacetabular impingement - the FAIR trial: a randomised controlled trial protocol. BMC Musculoskelet Disord 2014;15:58.

19 Harris-Hayes M, Holtzman GW, Earley JA, et al. Development and preliminary reliability testing of an assessment of patient independence in performing a treatment program: standardized scenarios. J Rehabil Med 2010;42:221-7.
20 Nilsdotter AK, Lohmander LS, Klässbo M, et al. Hip disability and osteoarthritis outcome score (HOOS)--validity and responsiveness in total hip replacement. BMC Musculoskelet Disord 2003;4:10.

21 Nilsdotter A, Bremander A. Measures of hip function and symptoms: Harris hip score (HHS), hip disability and osteoarthritis outcome score (HOOS), Oxford hip score (OHS), Lequesne index of severity for osteoarthritis of the hip (LISOH), and American Academy of orthopedic surgeons (AAOS) hip and knee questionnaire. Arthritis Care Res 2011;63 Suppl 11:S200-7.

22 Hinman RS, Dobson F, Takla A, et al. Which is the most useful patient-reported outcome in femoroacetabular impingement? test-retest reliability of six questionnaires. $\mathrm{Br} J$ Sports $\mathrm{Med}$ 2014;48:458-63.

$23 \mathrm{Kemp} \mathrm{JL}$, Collins NJ, Roos EM, et al. Psychometric properties of patient-reported outcome measures for hip arthroscopic surgery. Am J Sports Med 2013;41:2065-73.

24 Broderick JE, DeWitt EM, Rothrock N, et al. Advances in PatientReported Outcomes: The NIH PROMIS(®) Measures. EGEMS 2013;1:12.

25 Cella D, Riley W, Stone A, et al. The patient-reported outcomes measurement information system (PROMIS) developed and tested its first wave of adult self-reported health outcome item banks: 2005-2008. J Clin Epidemiol 2010;63:1179-94.

26 Bolton JE. Accuracy of recall of usual pain intensity in back pain patients. Pain 1999;83:533-9.

27 Vasiljevic D, Salsich GB, Snozek D, et al. Three dimensional kinematics of visually classified lower extremity movement patterns during a single leg squat among people with chronic hip joint pain. Physiother Theory Pract 2018:1-9.

28 Stratford PW, Balsor BE. A comparison of make and break tests using a hand-held dynamometer and the Kin-Com. J Orthop Sports Phys Ther 1994;19:28-32.

29 Bazett-Jones DM, Cobb SC, Joshi MN, et al. Normalizing hip muscle strength: establishing body-size-independent measurements. Arch Phys Med Rehabil 2011;92:76-82.

30 Koch GG, Tangen CM, Jung JW, et al. Issues for covariance analysis of dichotomous and ordered categorical data from randomized clinical trials and non-parametric strategies for addressing them. Stat Med 1998;17:1863-92.

31 OCEBM. Levels of Evidence Working Group"The Oxford Levels of Evidence 2". Oxford Centre for Evidence-Based Medicine. Available: https://www.cebm.net/index.aspx?o=5653 